\title{
XV.
}

\section{Proliferirendes Adenocystom der Mamma mit Flimmerepithelien.}

\author{
Von \\ Prof. Koloman Buday \\ in Klausenburg (Ungarn). \\ Hierzu Taf. VIII.
}

Es ist schon seit Langem bemerkt worden, dass die cystischen Geschwülste der Brustdrüse und des Eierstockes in ihrer histologischen Structur eine weitgehende Aehnlichkeit zeigen. Besonders die proliferirenden Adenocystome der genannten Organe sind sehr ähnlich gebaut; es kommen gutartige ebenso, wie maligne, papilläre und cystische Geschwülste in beiden Organen vor. Die Analogie wirả noch erhöht durch den Nachweis psammomartiger Concremente in den Eierstocks- und Mamma-Geschwülsten.

Auch die Zellen der papillären Cystadenome sind in beiden Organen ähnlich, cylindrisch, mit dem Unterschiede freilich, dass in den Ovarien öfters cystische Geschwülste mit Flimmerepithelien beobachtet werden, während in der Mamma Aehnliches - so weit ich weiss - nicht beschrieben wurde. Ich habe neulich ein proliferirendes Adenocystom der Mamma mit Flimmerepithelien zur Untersuchung bekommen; die Beschreibung dieses Falles dürfte von Interesse sein, weil die Analogie der Eierstocks- und Mamma-Geschwülste dadurch noch vollkommener wird. Ueberhaupt glaube ieh, dass die Thatsache, dass von einer ectodermalen Bildung, wie die Mamma, eine Neubildung mit Flimmerepithelien entstehen kann, constatirt $\mathrm{zu}$ werden verdient.

Der Fall ist anf der hiesigen chirurgischen Klinik des Prof. Brandt vorgekommen. Von der Krankengeschichte sei Folgendes hervorgehoben: 


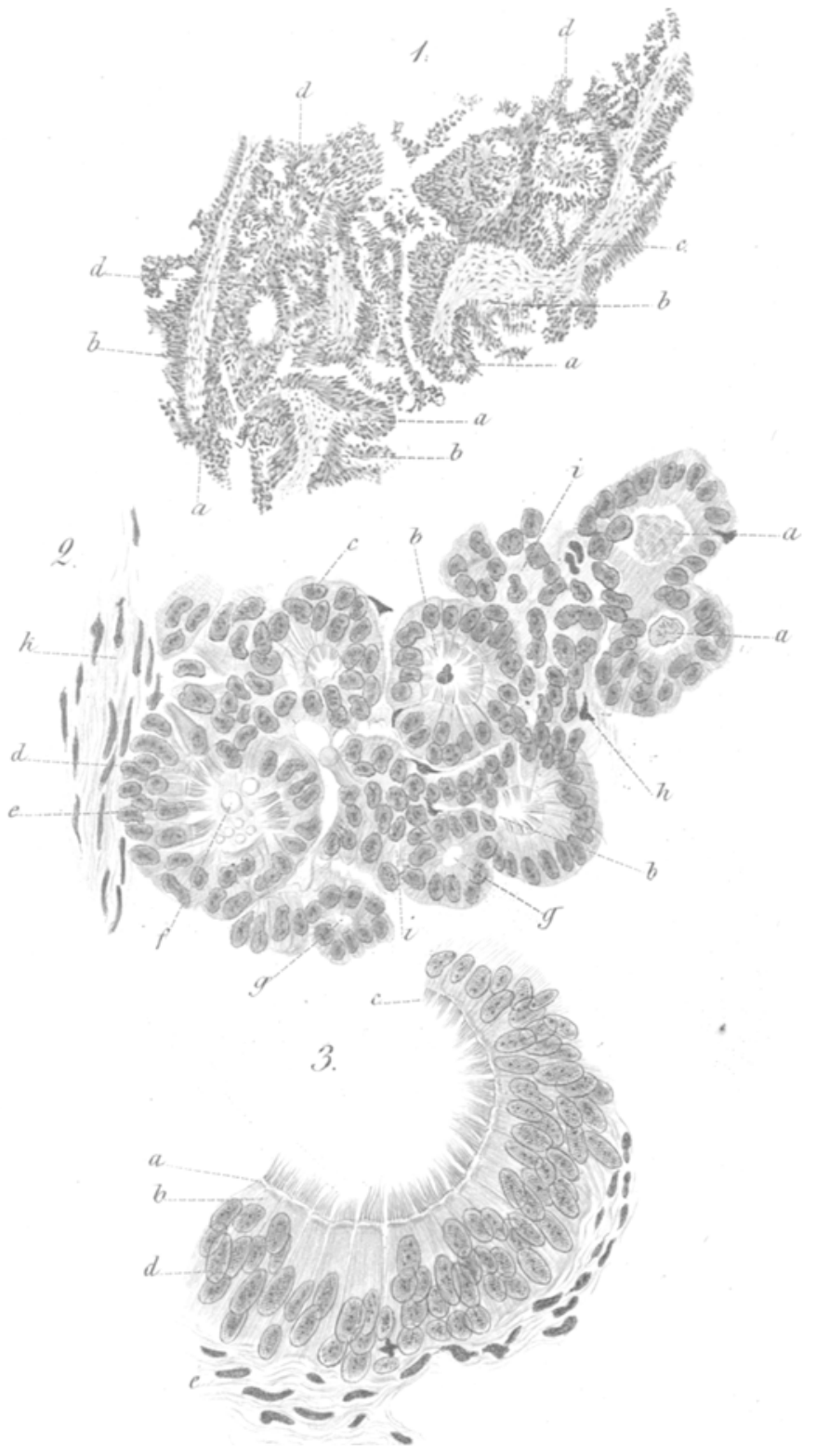


Josef Eisinkovich, 74 Jahr alt, Kaufmann, wurde am 17. August v. J. in das Krankenhaus aufgenommen; er soll früher, von Kopfschwerzen abgesehen, immer gesund gewesen sein. Seit einem Jahre bemerkte er in der rechten Brust einen stetig wachsenden, manchmal schmerzhaften Knoten.

Bei dem sonst wohl gebauten und gut genährten Manne findet sich in der rechten Mamma eine beinahe faustgrosse, scharfbegrenzte Geschwulst, welche sich äber der Thoraxwand leicht verschieben lässt und durch livide Haut bedeckt wird. Die Consistenz des Tumors ist ungleich, in der Mitte scheinbar fluctuirend, die Ränder mehr bart. Keine Achseldrüsen. Die klinische Diagnose lautete auf Sarkom.

Am 23. August wurde die Geschwulst, den leicht palpirbaren Grenzen entsprechend, mit der Brustwarze und der angrenzenden verdünnten Haut exstirpirt. Heilung per primam.

Auf dem Durchschnitte der Geschwulst seben wir eine beinahe gänseeigrosse Cyste, welche eine dicke, breiige, chokoladebraune Masse enthält. Die Wand der Cyste wird von der Haut bloss durch eine $1-2 \mathrm{~mm}$ dicke, fibröse Schicht getrennt, ihre Innenfläche ist ziemlich glatt; an einer Stelle ragt aber eine haselnussgrosse weisse Geschwulstmasse vor, welche scheinbar solid ist, bei der näheren Betrachtung jedoch aus feinsten, kaum sichtbaren - etwa mohnkorngrossen - Körnchen und Wärzehen zusammengesetzt erscheint. In der Nähe der Cyste, in das subcutane Fettgewebe eingebettet, liegt ein mehr als wallnussgrosser Geschwulstknoten, welcher von der. Umgebung scharf abgegrenzt ist, ohne eine besondere fibröse Kapsel zu baben. Dieser Knoten ist weich, markig-weiss, seine Schnittfäche jedoch etwas uneben, aus allerfeinsten Körnchen zusammengesetzt, welche in kleinen Spalten und Grübchen liegen und dieselben beinahe ganz ausfüllen. Die feingranulirte Schnittfläche ist an manchen Stellen einer mattgeschliffenen Glasscheibe nicht unähnlich. In der Geschwulstsubstanz sieht man ausser den feinen Körnchen und Spalten nur noch einige dickere Bindegewebsbalken, welche mit dem f́brösen Corpus der Brustdrüse zusammenhängen.

Den Inhalt der grossen Cyste bildeten geschrumpfte rotbe Blutkörperchei, Bjuipigmentkörnchen und Haematoidinkrystalle. Die mikroskopischen Schnitte der Cystenwand zeigen ausserdem noch einschichtige, njedrig-cubische Epithelzellen, welche der fibrösen, zellarmen Cystenwand innerlich anliegen, ohne eine continuirliche Schicht zu bilden; denn an vielen Stellen der Cyste ist kein Epithel nachweisbar. Offenbar sind die Zellen in Folge der Blutung zu Grunde gegangen.

Die weisse, markige Geschwulst-Substanz zeigt nach Formalin-Härtung bei der mikroskopiseben Untersuchnng einen sehr complicirten Bau, welcher am meisten einem stark proliferirenden Adenocystom entspricht. Man findet verschieden grosse Räume von sehr unregelmässiger Form, welche durch mebrschichtige Oylinderepithelien ausgekleidet sind. Regelmässige Cystchen mit glatter Wand finden sich nur vereinzelt; in den meisten erheben sich 
viele, sehr stark verästelte Papillen, welche in das Lumen hineinragen und dasselbe beinabe ganz ausfüllen.

Der sehr gracile Stiel, die grosse Länge und die starke Verästelung dieser dünnen Excrescenzen erklären es, dass in den centralen Theilen eines solchen Raumes die Quer- und Schrägsehnitte solcher Papillen isolirt nebeneinanderstehen, und weder mit einander, noch mit der Cystenwand zusammenzu hängen scheinen (Fig. 1).

An manchen Stellen sieht man auch bei schwächeren Vergrösserungen nichts als ein wirres Durcheinander dieser dichtgedrängten Papillen, welche das ganze Gesichtsfeld beherrschen.

Andere Theile sind weniger complicirt; die Wand der kleinen Cysten zeigt zwar auch hier papilläre Erhabenbeiten, aber diese sind niedriger und nicht so stark verästelt. Ueberall fällt es auf, dass das bindegewebige Stroma an der Bildung der Papillen sehr wenig betheiligt ist, so dass es den stark gewucherten Epithelien gegenüber ganz in den Hintergrund tritt. Die erste Anlage der Papillenbildung erfolgt durch eine Vermebrung der Epithelien, welche sich übereinander thürmen und eine-solide, manchmal stark in das Lumen hineinragende Epithelsprosse bilden. Einige erhalten alsdann eine sehr dünne, bindegewebige Axe, andere bleiben dauernd solid. Die Durchsebnitte solcher Epithelsprossen repräsentiren sich als kleine Epithelhäufchen, welche zwischen den wirklicben (d. h. eine bindegewebige Axe enthaltenden) Papillen sich finden (Fig. 1).

Das ohnehin complicirte Bild wird aber noch mehr verwiekelt, indem diese radiär verlaufenden Epithelsprossen stellenweise gegen das Centrum des Lumens durch tangentiale Fortsätze verbunden werden, wodurch tubulöse Räume mit ziemlich regelmässigem, rundlichem Lumen und radiär gestellten Cylinderzellen entstehen (Fig. 2, a, b, g).

Von einem gewöhnlichen tubulösen Bau, im Sinne tubulöser Drüsen, kann jedoch keine Rede sein, denn die Epithelzellen der nebeneinander liegenden Tubuli bilden einen zusammenbängenden Complex, $d$. $h$. sie confluiren mit einander, so dass von einem bindegewebigen Stroma, welches die scheinbaren Tubuli umgeben und von einander trennen würde, beinahe nichts zu sehen ist (Fig. 1).

Die langen, anastomosirenden Epithelreihen bilden ausserdem plexiforme Stränge, welche ebenfalls durch den Mangel eines bindegewebigen Stromas auffällen (Fig. 1, d).

Je nach der versehiedenen Art der Zusammenfügung der Zellreihen wechselt auch die Form der Zellen nicht unbeträchtlich. Diø dem fibrösen Stroma zunächst liegenden sind hocheylindrisch, die Zellen jener Epitbelbäufchen dagegen, welche kein bindegewebiges Stroma enthalten, niedriger, cubisch oder polymorph. Eine Differenzirung der Żellen in einen proximalen und einen distalen Theil, wie in den cylindrischen Zellen, ist nicht zu bemerken, der Kern liegt in der Mitte (Fig. 2, i). Im Allgemeinen ist der Epithelbelag der Cysten und Papillen ziemlich ungleichmässig, bald 
zweizeilig, bald zwei- bis vierschichtig. Kerntheilungs-Figuren finden sich in beschränkter Zahl überall, sie weisen nichts Abnormes auf.

Die tubulösen Räume, welche die Geschwulstzellen einschliessen, sind entweder ganz leer, oder durch eine homogene, colloidartige Masse ausgefüllt. (Fig. 2, a); manche enthalten kugelförmige, schleimartige Tröpfchen, welche die Secretions-Producte der Zellen darstellen.

Das Stroma ist, von einigen breiteren Septen abgesehen, sehr gering, die meisten Papillen laben nur eine sebr dünne, kaum wahrnehmbare, fibröse Axe; nur ausnahmsweise finden sich Papillen mit deutlichem Stroma, welches zuweilen schleimig oder byalin degenerirt ist. Papillen mit völlig byalinem, breitem Stroma zeigen ein eigenthümliches Bild, wie es auch in Ovarialtumoren öfters vorkommt. Der tubulöse Bau der Epithelhäufchen ist am meisten in der Nähe der breiteren Bindegewebszüge ausgeprägt; eine homogene Membrana propria, wie bei den Milchgängen der normalen Milchdrüse, jst nirgends sichtbar, ebensowenig bemerkt man eine pericanaliculäre, concentrische Anordnung der Bindegewebsfasern.

Die merkwürdigste Erscheinung des mikroskopischen Befundes ist der deutliche Flimmerbesatz bei einer grossen Anzahl der Epithelzellen. Solche Zellen kommen zwar nicht überall in gleicher Zahl, aber in fast allen Theilen der Geschwulst vor.

An den bervorragendsten Stellen der Papillen sieht man nur vereinzelte Flimmerepithelien; um so zahlreicher sind sie in dem Recessus zwischen den Papillen, wo sie eine fast ununterbrochene Reibe bilden (Fig. 3). Die Stränge cubischer oder polymorpher Zellen enthalten fast gar keine Flimmerepithelien, immer sind es die mehr oder weniger hoben Cylinderzellen, welche Flimmern aufweisen.

Es braucht kaum hervorgeboben zu werden, dass die Zellen mit ihren Flimmern immer gegen ein Lumen gerichtet sind, welches manchmal unregelmässig und gross, manchmal dagegen äusserst klein ist. - Was die feinere Structur der Flimmerepithelien betrifft, so fällt an den meist bohen Cylinderzellen eine sehr ausgesprochene Längsstreifung auf, wie es von verschiedener Seite (Eberth, EngeImann, Nussbaum) von den Flimmerepithelien beschrieben wurde (Fig. 3, b). Diese Iängsstreifen finden sich in dem distal vom Kerne liegenden Theil der Zelle und scheinen in den Flimmerhärchen ihre Fortselzung zu finden; die Flimmern halten jedoch nicht die Richtung der Zellstreifen ein, sondern bilden einen spitzen Winkel mit denselben, so dass die Härchen auf dem Rande der Zellen zumeist scbief gestellt sind.

Zwischen dem Flimmerbesatz und dem gestreiften Theile der Zelle liegt ein sebr deutlicher, horizontaler Basalsaum, unter welchem nocb sich ein dunkler Streifen befindet, der sogenannten Knötehenzone entsprechend, während zwischen den zwei dunklen Zonen eine hellere, intermediöre Schicht liegt (Fig. 3, a).

Ausser diesen, mit ganz typischen Wimperhaaren versehenen Zellen finden sich auch solche, welche zwar einen gut ausgesprochenen Saum 
zeigen, aber an Stelle der Härchen nur einen homogenen, stumpf-kegelförmigen Fortsatz aufweisen, in welchem keine Härchen differenzirt sind (Fig. 3, c). Sie bilden, so zu sagen, die unentwickelten Formen, d. i. den Uebergang einfacher Cylinderzellen in die typischen Flimmerepithelien. Besonders die niedrigen Cylinderzellen der kleineren tubulösen Räume lassen öfters solebe abortive Formen der Flimmerhaare bemerken. Man sieht auch ganz kleine Tubuli mit kleinen, zum Theil noch unausgebildeten Flimmerepithelien ausgekleidet.

Einige der Flimmerepithelien zeigen typische Mitosen, wobei die Axe der Kernspindel mit der Längsaxe einen Winkel von $90^{\circ}$ bildet.

Der Bau der Flimmerzellen erinnert sebr an die Flimmerepithelien der Trachea, auch was die zweizeilige Lage der Zellen betrifft; denn auch in unserem Falle waren die Zellen dort, wo Flimmerbärchen vorkamen, meist zweizeilig, nicht mehrschichtig, wie zumeist an den wimperlosen Stellen. Ueberhaupt geht mit der Flimmerbildung eine gewisse Regelmässigkeit der Form und der Ordnung der Zellen parallel, denn die meisten Flimmerepitbelien sind hohe Cylinderzellen, welche beinabe gleich gross sind und eine gleichmässige Reihe bilden.

Die Genese der Flimmerzellen lässt sich so auffassen, dass die auskleidenden Cylinderzellen eines grösseren Raumes sich zuerst in Form solider Zapfen anbäufen; diese Papillen bilden alsdann, mit einander confluirend, einen netzförmigen Zellenplexus mit dazwischen liegenden kleinen, tubulösen Räumen. Jene Zellen, welche solche Räume einschliessen, ordnen sich radiär, bilden oft eine colloide Masse, und enthalten an ibrem gegen das Lumen gerichteten Rande zuerst einen breiten Saum mit einem homogenen Fortsatz, später aber wirkliche Flimmerbärchen (S. Fig. 2).

In der Nähe der Geschwulst sieht man in dem fibrösen Körper der Mamma einige Milchgänge mit mässig erweitertem Lumen, zweischichtigem Cylinderepitbel und angehäuftem, aus zerfallenen Zellen bestehendem Inhalt. Sonst enthalten die Milchgänge keine Uebergangsbilder zur Geschwulst, überhaupt keine Proliferations-Erscheinungen. Normale Acini der Milchdrüse habe jeh nicht gefunden.

Wie aus dem mikroskopischen Befunde hervorgeht, handelt es sich in diesem Falle um eine adenomatöse Geschwulst, in welcher die Stromabildung durch die mächtige Proliferation der Epithelien völlig in den Hintergrund gestellt wird. MammaGeschwülste von adenomatösem Bau und mit reichlichen papillären Excrescenzen sind zwar nicht häufig, doch sind sie öfters beschrieben worden; merkwürdigerweise fanden sie sich meistens bei älteren Individuen vor. So ist z. B. bei Leser ${ }^{1}$ ) ein ähn-

(1) Beitrag zur pathologischen Anatomie der Gesehwülste der Brustdrüse. Ziegler's Beiträge z. path. Anatomie II. 
licher Fall beschrieben, in welchem, ebenfalls bei einem Manne, die Entwicklung aus den Milchgängen nachzuweisen war.

Es ist wahrscheinlich, dass auch in unserem Falle den Ausgangspunkt der Geschwulstbildung die Milchgänge bildeten, obwohl die mikroskopische Untersuchung dafür keine Anhaltspunkte gab. Bei Geschwülsten, welche aus circumscriptem, kleinem Gebiet hervorgehen, ist eben, besonders in späteren Stadien, nicht viel von der ersten Entwickelung festzustellen.

Die Dürftigkeit der Stromabildung zeigt zur Genüge, dass unser Fall von den intracanaliculären Fibromen und Sarkomen sich ganz wesentlich unterscheidet und den proliferirenden Cystadenomen anzureihen ist, deren Aehnlichkeit mit den analogen Geschwülsten der Eierstöcke schon in den einleitenden Worten hervorgehoben wurde.

Sasse ${ }^{1}$ ), welcher neuestens die cystischen Tumoren der Mamma einer eingehenden Untersuchung unterzog, unterscheidet zwei Typen der papillären Cystadenome:

1. Das intracanaliculäre Cystadenom, mit einem dichten Netzwerk zahlreicher, mit einander communicirender, bald enger, bald weiterer, unregelmässig ausgebildeter Drüsenschläuche, ohne eigentliche Papillenbildung. Die Drüsenschläuche enthalten meist einschichtiges Cylinderepithel, die Geschwülste sind sonst gutartig.

2. Cystadenoma proliferum destruens malignum. Sie sind den proliferirenden Ovarialcysten ähnlich; die papillären Excrescenzen sind mit mehrschichtigem, manchmal hundertfachem Epithel bedeckt. Man findet auch solide Zellennester; überhaupt geht die Geschwulst oft in Krebs über.

Von diesen zwei Typen entspricht zwar der zweite mehr unserem Falle, als der erstere, denn die Epithelıen sind zumeist mehrschichtig; auch die wechselnde, bald cylindrische, bald cubische oder polymorphe Gestalt der Zellen ist meistens ein Zeichen der Malignität' der cystischen Geschwülste. Es wäre dennoch verfehlt, die beschriebene Geschwulst als eine ausgesprochen maligne bezeichnen zu wollen, denn es waren weder solide Krebsnester, noch die Zeichen eines unbeschränkten, infil-

1) Ueber Cysten und cystische Tumoren der Mamma. Arehiv für klinische Chirurgie 54 . 


\section{1}

trativen Wachsthumes nachzuweisen. Auch klinisoh repräsentirte sich die Geschwulst eher als ein Sarkom, d. i. als eine, durch expansives Wachsthum sich vergrössernde Neubildung. Trotz der entgegenlautenden Befunde Pfannenstiels ${ }^{1}$ ) glauben wir; dass es manchmal recht schwer ist, zwischen Adenomen und Carcinomen eine feste Grenze zu ziehen. - Auch hier haben wir es mit einem Adenom $\mathrm{zu}$ thun, welches einige Zeichen der Malignität zeigt, ohne dass alle Kriterien einer carcinomatösen Degeneration nachzuweisen wären.

Besonders interessant sind jene Stellen der Geschwulst, wo die tubulöse Structur bloss durch die radiäre Gruppirang der Epithelzellen, ohne Beihülfe des beinahe völlig fehlenden Stromas, entsteht. Sasse beschreibt ein ähnliches, tubulöses Adenom ohne Stromabildung (XXI. Fall l. c.).

Noch merkwürdiger ist unser Fall durch die Bildung zahlreicher Flimmerzellen. Eine Beweglichkeit der Flimmerhärchen wurde nicht beobachtet, da die Geschwulst erst nach FormalinHärtung untersucht wurde. Doch ist es zweifellos, dass es sich wirklich um Flimmerepithelien, und nicht etwa um Stäbchenzellen, handelt, wie sie z. B. im Darm vorkommen, denn die Härchen sind in gut ausgebildeten Flimmerzellen unseres Falles sehr lang, sie stehen ganz frei neben einander, und gehen in einen sehr deutlich entwickelten Saum mit einer gut ausgebildeten Knötchenzone über; auch die Streifenzone (der intracelluläre Fadenapparat) ist leicht zu unterscheiden.

Die Beweglichkeit der Härchen wird sehr wahrscheinlich gemacht durch den Umstand, dass dieselben auf dem Basalsaume schief stehen; denn die Gleichgewichtslage der Härchen weicht meistens von der auf die Oberfläche der Zellen verticalen ab.

Jene Zellen, in welchen der Saum homogen ist oder keine deutlichen Härchen unterscheiden lässt, fasse ich als unentwickelte Flimmerepithelien auf, welche erst später zur vollen Differencirung der Härchen gekommen wären. Es ist bekannt, dass unter den Stäbchenzellen des Darmes, ausser den Zellen mit ge-

1) Die Genese der Flimmerepithelgeschwülste des Eierstockes. Archiv für Gynaek. 40. 
streiftem Saum, auch solche mit homogenem vorkommen; vielleicht werden durch unsere Zellen mit homogenem Saum auch solche Vorstufen der Flimmerepithelien dargestellt. Dagegen scheint allerdings jene allgemeine Ansicht zu sprechen, wonach die Flimmerhärchen aus dem Basalsaume hervorsprossen; doch ist die Genese der Flimmerzellen noch nicht hinlänglich bekannt, so dass wir nicht ausschliessen können, dass sie auch durch Differenzirung in einer homogenen Schicht entstehen. In Folge dessen halte ich den homogenen Fortsatz dieser Zellen nicht für eine Degeneration, sondern für eine unvollständige Entwickelung des Flimmerbesatzes.

Wollen wir die Bedeutung der Flimmerepithelien in unserem Falle feststellen, so müssen wir zuerst kurz das Vorkommen der Flimmerzellen in pathologischen Verhältnissen besprechen.

Man kann die beschriebenen Fälle in zwei Gruppen eintheilen. In einer Reihe der Fälle handelte es sich um kleine, meist uniloculäre Cysten, welche mit Flimmerepithelien ausgekleidet waren, und keinen Geschwulstcharakter, d. i. keine grössere Wachsthumsenergie, zeigten. Solche Cysten sind am Halse, Oesophagus, an der Pleura, Leber etc. öfters gefunden worden. (S. die Literatur bei Zahn.) ${ }^{\mathbf{1}}$ )

Die meisten dieser Fälle lassen sich durch die Annahme embryonal verlagerter Keime erklären, sie sind also sogenannte teratoide Cysten.

Die andere Gruppe umfasst multiloculäre Cysten mit ausgesprochen geschwulstartigem Charakter, mit Neigung zu unbeschränktem Wachsthum. Solche cystischen Geschwülste mit Flimmerepithelien kommen oft in den Ovarien vor, seltener wurden sie anderswo beobachtet. So z. B. beschrieb Virchow ${ }^{2}$ ) einen Mediastinaltumor mit Cysten, welche theils mit Plattenepithel, theils mit Flimmerepithel ausgekleidet waren; die Bösartigkeit dieses Tumors war durch Metastasenbildung orwiesen. Der angeführte Fall war ebenfalls eine teratoide Geschwulst, während von den Ovarialcysten mit Flimmerzellen eine solche

1) Ueber mit Flimmerepithelien ausgekleidete Cysten. Dieses Archiv 143.

2) Teratoma myxomatodes mediastini. Dieses Archiv 53. 
Genese nicht erwiesen, sogar nach den Untersuchungen Pfannenstiel's unwahrscheinlich ist.

Ich bin der Meinung, dass auch in unserem Falle die Entstehung von Flimmerzellen in einer Mammageschwulst nicht nnbedingt für einen teratoiden Ursprung spricht. Man ist allerdings gewöhnt, in allen solchen Fällen eine Verlagerung von embryonalen Keimen anzunehmen. So z. B. schrieb Waldeyer von den flimmernden 0varialcysten: „Ich möchte es also unentschieden lassen, ob das Flimmerepithel sich in echten Ovarialcysten anders, als bei teratoiden Bildungen vorfindet. Wenigstens scheint sonst Flimmerepithel pathologisch nur in teratoiden Geschwülsten oder an Localitäten vorzukommen, wohin es auch normal gehört."

Seitdem ist aber, u.A. durch die Untersuchungen Pfannenstiel's, wahrscheinlich geworden, dass die flimmernden Ovarialcysten im späteren Lebensalter durch die Einsenkung des flimmerlosen Keimepithels in das Ovarium entstehen. Es kann also angenommen werden, dass ein sonst nicht flimmerndes Epithel im extrauterinen Leben unter Umständen sich in ein flimmerndes verwandeln kann. Aehnliches kommt nach v. Recklinghausen bei der Ranula, an den sonst nicht flimmernden Zellen der Schleimgänge, vor. Die Ursache dieser Umwandlung sucht v. Recklinghausen in besonderen Ernährungsverhältnissen, indem er sagt: „Der Wechsel in der Beschaffenheit der Epithelzellen hängt von den Zuständen der Unterlage ab, speciell von der Ernährung, welche ihnen durch die Grösse der Blutströmung geboten wird " ${ }^{1}$ ).

Es ist nicht unmöglich, dass auch in unserem Falle die Geschwulst nicht aus verlagerten embryonalen Keimen entstand, umsomehr, als das hohe Alter des Patienten die Entwickelung aus congenitaler Anlage unwahrscheinlich macht. Will man den Fall als einen teratoiden auffassen, so stösst man auf Schwierigkeiten, denn es wäre schwer zu sagen, woher die Flimmerzellen stammen, da in der normalen Mamma in keinem Stadium der Entwicklung Flimmerzellen vorhanden sind.

Für viel ungezwungener halte ich die Erklärung, nach welcher in der Geschwulst die veränderten Ernährungsverhältnisse,

1) Ueber die Ranula u. s.w. Dieses Archiv 84. 
und noch mehr die formativen Reize, die bei der Geschwulstbildung eine grosse Rolle spielen, eine Metaplasie des Cylinderepithels zu Flimmerepithel veranlassten. Dass so etwas in der Mamma vorkommen kann, wäre um so weniger zu bewundern, als die Mamma den Sitz der mañnigfaltigsten Bindesubstanzund epithelialen Geschwülste bildet.

Die eigentliche Ursache der Geschwulstbildung entzieht sich auch hier, wie in den meisten Fällen, der Beurtheilung. Dass die cystischen Geschwülste mit Flimmerepithelien nicht immer gutartig sind, das beweisen, ausser dem erwähnten Falle Virchow's, auch mehrere Eälle von flimmernden Ovarialkystomen, welche mit Carcinom combinirt waren; Pfannenstiel erwähnt auch einen Fall. Ich selbst sah unlängst bei einem Adenocarcinom der Gebärmutter ausser typischen Krebsnestern auch solche, welche mit Flimmerepithelien ausgekleidete Räume zeigten.

Es ist nicht auszuschliessen, dass auch unser Fall, trotz der Flimmerepithelien, das erste Stadium einer carcinomatösen Entartung darstellte, da derselbe, wie gesagt, mehrere Zeichen von Malignität verrieth.

\section{Erklärung der Tafol VIII.}

Fig. 1. Aus der Mitte einer grösseren Cyste. a) Mebrschichtiges Cylinderepithel; b) Stroma der Papillen; c) Epitbel mit Flimmerbärchen; d) netzförmige Epithelstränge, ohne Stromabildung. Nach photogr. Aufnahme. Vergr. etwa 60.

Fig. 2. Tubulöse Räume obne Stromabbildung. a) Tubuli mit Colloidpfröpfen; b) Zellschläuche mit Flimmerepithelien; c) dieselben mit homogenem Fortsatz der Epithelien; d) Saum der Flimmerzellen; e) Flimmerbärchen; f) Schleimtröpfchen; g) kleine Zellschläuche; b) Bindegewebszellen des Stroma; i) Zellstränge mit den Zellschläuchen zusammenhängend; k) Stroma. Reich. Obj. VI. Oc. IV.

Fig. 3. Flimmerepithelien eines tubulösen Raumes. a) Saum mit der Knötchenzone; b) intracelluläre Fäden; c) Flimmern; d) Kerne der Flimmerzellen; e) Stroma. Imm. $\frac{1}{12}$ Oc. II. 